\title{
Yeast ER-Golgi v-SNAREs Bos1p and Bet1p differ in steady-state localization
}

\section{and targeting}

\author{
Dmitri Ossipov, Stephan Schröder-Köhne and Hans Dieter Schmitt* \\ Department of Molecular Genetics, Max-Planck-Institute for Biophysical Chemistry, D-37070 Göttingen, Germany \\ *Author for correspondence
}

Accepted 1 September; published on WWW 3 November 1999

\section{SUMMARY}

Vesicle specific SNAP receptors ( $v$-SNAREs) Bos1p and Bet1p are involved in targeting of anterograde vesicles between the endoplasmic reticulum (ER) and early Golgi of Saccharomyces cerevisiae. To analyze factors that influence the targeting of these proteins, $\alpha$-factor tagged versions of Bos1p and Bet1p were employed. The $\alpha$-factor can be cleaved off by the Kex2p protease as soon as the hybrid proteins reach the late Golgi compartment. The data obtained by monitoring of Kex2p cleavage, by immunofluorescence microscopy and cell fractionation showed that Bos1- $\alpha$ and Bet1- $\alpha$ have different cellular localization and dynamics. Bos1- $\alpha$ is an ER protein, which recycles between the Golgi and the ER in COPI-dependent manner. Bet1- $\alpha$ is an early Golgi protein and it does not change its localization under conditions when other recycling Golgi proteins can be trapped in the ER.

Key words: Bet1p, Bos1p, ER-Golgi, v-SNARE, Retrograde transport, COPI

\section{INTRODUCTION}

The machineries that mediate the bidirectional transport between the endoplasmic reticulum (ER) and Golgi are conserved between yeast and higher eukaryotes (Schekman and Orci, 1996; Hong 1998). There are two types of coated vesicles which mediate that transport, COPI and COPII (Waters et al., 1991; Barlowe et al., 1994; Duden et al., 1994; Letourneur et al., 1994; Aridor et al., 1995). COPII vesicles are carriers for the anterograde transport from the ER to the Golgi (Bednarek et al., 1995). They were first identified in yeast but homologues were found in mammalian cells as well (Barlowe et al., 1994; Aridor et al., 1995). The COPII coat includes 4 subunits and the GTPase Sar1p. COPII is implicated in vesicle budding and cargo selection (Kuehn et al., 1998; Aridor et al., 1998).

Coatomer is a protein complex consisting of 7 subunits which forms the coat of COPI vesicles in mammalian as well as in yeast cells. In addition, the coat of COPI vesicles contains the small GTPase Arf1p (Waters et al., 1991; Duden et al., 1994, 1998; Stamnes et al., 1998). These coat proteins are involved in the formation of vesicles as well as cargo selection. COPI vesicles may function both in retrograde transport and anterograde transport (Letourneur et al., 1994; Bednarek et al., 1995; Rowe et al., 1996; Gaynor and Emr, 1997; Orci et al., 1997; Scales et al., 1997; Spang et al., 1998; Stamnes et al., 1998). There is strong evidence that COPI vesicles are involved in the recycling of proteins from the Golgi back to the ER. Type I transmembrane proteins of the ER carrying a C-terminal di-lysine retrieval signal fail to be retained in the ER in retl-1 and $\sec 27-1$ ( $\alpha$-COP, $\beta$ -
COP) mutants as well as in mutants with defects in other coatomer subunits $(\sec 21-1, \sec 21-2, \operatorname{ret} 2-1, \operatorname{ret} 3-1=\gamma-, \delta$ and $\zeta$-COP) (Letourneur et al., 1994; Cosson et al., 1996). Some receptors or sorting factors as well as their ligands and substrates also depend on coatomer for proper dynamic localization: Erd2p, a receptor required for retrograde transport of soluble ER-resident proteins carrying HDEL signal (Lewis and Pelham, 1996); Rer1p, which mediates retrieval of ER-resident transmembrane proteins lacking a di-lysine signal (Boehm et al., 1997; Sato et al., 1997); Sec22p, which acts as a vesicle-specific receptor on ER- and Golgi-derived vesicles (Søgaard et al., 1994; Lewis et al., 1997; Ballensiefen et al., 1998).

It is believed that coated vesicles loose their coat before they dock and fuse to the target compartment (Rothman, 1994). A set of specific membrane receptors is required for fusion. Receptors exposed on the vesicle membrane ( $v$-SNAREs) can interact with $t$-SNAREs, receptors situated on the membrane of the target compartment (Söllner et al., 1993; Søgaard et al., 1994). According to recent data, both types of receptors have to be activated by the ATPase NSF ( $N$-ethylmaleimidesensitive factor) before this interaction can take place (Mayer et al., 1996; Otto et al., 1997). Although there is a certain promiscuity of SNARE proteins, in general each step of the vesicular transport involves distinct subsets of $v$ - and $t$ SNAREs (Söllner and Rothman, 1994; Götte and Fischer von Mollard, 1998; Yang et al., 1999).

There are several $v$-SNAREs required for ER-Golgi transport in yeast. They are Bos1p, Bet1(Sly12)p, Sec22(Sly2)p and Ykt6p (Dascher et al., 1991; Newman et al., 1992; Søgaard et al., 1994). Sed5p is known to be a $t$-SNARE 
for the anterograde transport step (Hardwick and Pelham, 1992). Retrograde transport of COPI vesicles involves the $t$ SNARE Ufe1p (Lewis and Pelham, 1996) located in the ER membrane and the Sec20p/Tip20p complex (Sweet and Pelham, 1992; Lewis et al., 1997). As Sec22p is found in a SNARE-complex with Ufe1p it may be a $v$-SNARE for the retrograde transport in addition to its proposed role in anterograde transport (Lewis et al., 1997).

In this study we analyzed the targeting of the ER-Golgi $v$-SNAREs Bos $1 \mathrm{p}$ and Bet $1 \mathrm{p}$. Recombinant versions of the $v$-SNAREs tagged with protease-cleavable epitopes were constructed to trace the cellular fate of these transmembrane proteins. Processing by the Kex $2 p$ protease and detection of cleaved and uncleaved constructs by western blotting analysis combined with immunofluorescence experiments allowed us to monitor the passage of the hybrid proteins through compartments of the early secretory pathway. These experiments have shown that Bos $1 \mathrm{p}$ and Betlp occupy different subcellular compartments at steady state and differ with respect to their dynamic targeting properties.

\section{MATERIALS AND METHODS}

\section{Growth conditions and yeast strains}

If not indicated otherwise Saccharomyces cerevisiae cells were grown in synthetic minimal medium containing galactose $(2 \%)$ or glucose (2\%) as carbon sources and supplemented as necessary with $20 \mathrm{mg} / \mathrm{l}$ tryptophan, histidine, adenine or uracil, or $30 \mathrm{mg} / \mathrm{l}$ leucine or lysine. To ensure that pairs of isogenic mutant and wild-type cells were used, comparisons of COPI-mutants (sec21-1 and sec27-1) with the corresponding wild-type cells were carried out using mutant cells transformed with either a single copy plasmid encoding the particular wild-type protein or the same vector without insert (pRS315-SEC21, pRS315-SEC27, or pRS315 alone. For cloning purposes Escherichia coli RRI was used. Saccharomyces cerevisiae strains used in this

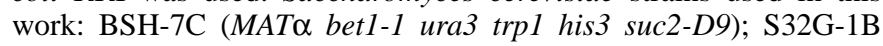
(MAT人 sec32-1/(bos1) ura3 leu2 GAL); S21P4-6A (MATa leu2 ura3 lys2 pep4::HIS3); S21P4-9A (MATa sec21-1 leu2 ura3 pep4::HIS3);

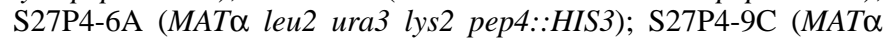

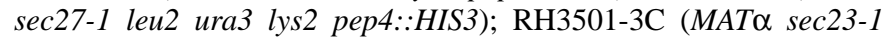
leu2 ura3); SSY78 (MATa sec23-1 trp1 his ura3 leu2 mycEMP47::LEU2 pHA-PMR1::URA3).

\section{Construction of the BOS1/BET1 $\alpha$-factor hybrid genes}

The $\alpha$-factor-tagged Bos $1 \mathrm{p}$ and Bet1p proteins were constructed as follows. Plasmid pWB-GALAC $\alpha$ (Ballensiefen et al., 1998) was cleaved with $N c o$ I and $S m a$ I to obtain a $1.1 \mathrm{~kb}$ fragment including a part of the SEC22 gene carrying the transmembrane domain encoding segment of of SEC12, a $c-m y c$ tag and $\alpha$-factor sequences (including the last 13 codons plus Kex2 cleavage site and $0.3 \mathrm{~kb}$ of 3'nontranslated sequences). This fragment was cloned into the pYX113 vector (R\&D Systems, Minneapolis) downstream of the GAL1 promoter. To remove the rest of SEC22 and SEC12 sequences this plasmid was cleaved with $\mathrm{NcoI}$ and BamHI. BOS1 and BET1 were amplified by PCR using primers with linker regions carrying $N c o$ I and $B a m H I$ restriction sites and then inserted into the latter plasmid producing DO-1 (ARS/CEN, URA3, GAL1-BOS1-c-myc- $\alpha$ ) and DO2 (ARS/CEN, URA3, GAL1-BET1-c-myc- $\alpha$ ), respectively. pYX113BET1-HA (ARS/CEN, URA3, GAL1-BET1-HA) was obtained by cloning Bet1p derived from DO-2 into pYX113 using EcoRI and BamHI sites. For double immunofluorescence plasmid $\mathrm{pJOCH}$ ( $2 \mu, U R A 3, T P I-O C H 1-c-m y c$; kindly provided by Mike Lewis, Cambridge, UK) was used.

\section{Antibodies}

The monoclonal anti-c-myc antibody 9E10 (Evan et al., 1985), the polyclonal anti-c-myc antibody (A-14) and polyclonal anti-HA antibody (Y-11) were obtained from Santa Cruz Biotechnology. Antibodies against Rer1p (J. Boehm), and $\alpha$-factor (M. Benli and D. Gallwitz) were raised in rabbits. The polyclonal serum against Emp47p (AL) has been described (Schröder et al., 1995). For ECLdetection of the hybrid proteins, HRP-coupled secondary anti-rabbit or anti-mouse antibodies were purchased from Jackson Laboratories. Cyanine-(Cy2 or Cy3)-conjugated secondary antibodies were from Amersham and Jackson Laboratories, respectively.

\section{Protein extraction and immunoblotting}

Western blotting analysis was performed as described by Boehm et al. (1994). Aliquots $\left(1 \mathrm{OD}_{600}=1.7 \times 10^{7}\right.$ cells $)$ of transformed cells were lysed in $2 \mathrm{M} \mathrm{NaOH}, 5 \% \quad \beta$-mercaptoethanol and proteins precipitated with $10 \%$ trichloroacetic acid (TCA), neutralized with 1 M Tris-base and dissolved in SDS sample buffer. Proteins were resolved on $12.5 \%$ SDS-PAGE. To re-use the nitrocellulose filters for different immunoblot assays, they were stripped for 30 to 60 minutes with $0.2 \mathrm{M}$ glycine, $\mathrm{pH} 2.5$.

\section{Immunoprecipitation}

Immunoprecipitation using anti-c-myc and anti- $\alpha$-factor antibodies was performed as described by Boehm et al. (1994).

\section{Indirect immunofluorescence}

Immunofluorescence was performed as described by Schröder et al. (1995). Cells expressing the GAL1-controlled SEC22- $\alpha$ gene were grown overnight in selective minimal medium containing galactose to reach $\mathrm{OD}_{600}$ 1-1.5 (pulse conditions). An equivalent of $10 \mathrm{OD}_{600}$ of cells was collected and resuspended in $10 \mathrm{ml}$ glucose-containing selective medium (chase conditions). Alternatively, in some experiments, cells were treated with cycloheximide $(20 \mu \mathrm{g} / \mathrm{ml})$ to stop protein synthesis. After a chase time of 2 hours the cells were prefixed by adding $1.3 \mathrm{ml}$ of $37 \%$ formaldehyde. After one hour at room temperature fixation was continued in $1 \mathrm{ml}$ of a paraformaldehyde solution for two additional hours $(3.5 \%$ paraformaldehyde in PBS/10\% sorbitol). The cells were then washed with PBS/10\% sorbitol, resuspended in $1 \mathrm{ml}$ of this buffer and treated with 60 units zymolase in the presence of $5 \mu \mathrm{l} / \mathrm{ml} \beta$-mercaptoethanol for one hour to remove cell walls. Spheroplasts were collected, washed four times in PBS/10\% sorbitol and resuspended in $400 \mu \mathrm{l}$ of PBS/10\% sorbitol. $15 \mu \mathrm{l}$ of this suspension was applied per slide well, previously treated with a poly-lysine solution (Sigma) to allow cell attachment. After 10 minutes the suspension was removed and $15 \mu \mathrm{l}$ of blocking solution (PBS/10\% sorbitol, 1\% Triton X-100, 1\% milk powder) applied for 10 minutes. Incubation with the first antibody (A-14, 9E10) was carried out overnight at $4{ }^{\circ} \mathrm{C}$ at appropriate dilution in blocking solution. After seven consecutive washes of these cells with PBS/10\% sorbitol, secondary antibody was added (Cy3-conjugated Fab fragment of goat anti-rabbit or anti-mouse IgG, Cy2-conjugated antirabbit or anti-mouse $\mathrm{IgG}$ ). After 1 hour at $30^{\circ} \mathrm{C}$ the cells were washed seven times with $\mathrm{PBS} / 10 \%$ sorbitol solution, DNA was stained with DAPI (4',6-diamidino-2-phenylindole), and cells were washed twice in PBS $/ 10 \%$ sorbitol. Embedding medium was applied to the slides which were then sealed with a coverslip.

\section{Subcellular fractionation by velocity sedimentation on sucrose-density gradients}

Cells were grown overnight at $25^{\circ} \mathrm{C}$ to $\mathrm{OD}_{600} 0.35$ in PM-glucose medium (2\% glucose, $0.5 \%$ Gibco-peptone 140, 0.5\% ammonium sulfate, $0.17 \%$ Difco-yeast nitrogen base). $600 \mathrm{ml}$ of the culture were harvested and cell were resuspended in $100 \mathrm{ml}$ PM-glucose supplemented with $20 \mu \mathrm{g} / \mathrm{ml}$ cycloheximide. The culture was split into two aliquots, one of which was kept shaking at $25^{\circ} \mathrm{C}$. The other aliquot was incubated in a shaking water bath at $35^{\circ} \mathrm{C}$. After one hour 
cells were harvested and energy-production was stopped by resuspending the cells in $10 \mathrm{ml}$ buffer A (10 mM Hepes, pH 7.5, 1 $\mathrm{mM} \mathrm{MgCl} 2,1 \mathrm{M}$ sorbitol, $10 \mathrm{mM} \mathrm{NaN}_{3}, 10 \mathrm{mM} \mathrm{NaF}, 20 \mu \mathrm{g} / \mathrm{ml}$ cycloheximide). Sodium $\beta$-mercaptoethanesulfonate (MESNA) was added to $100 \mathrm{mM}$ to reduce cell walls. After 10 minutes cells were harvested again and washed with $10 \mathrm{ml}$ buffer A. The cell-pellet was taken up in $1 \mathrm{ml}$ buffer A. Recombinant $\beta$ 1,3 glucanase (Shen et al., 1991) was added to spheroplast the cells. After 30 minutes of incubation at $30^{\circ} \mathrm{C}$ cells were harvested and osmotically lysed by

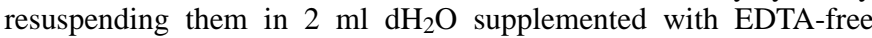
protease inhibitor mix (Boehringer Mannheim). The lysis efficiency was about $80 \%$. The lysates were cleared from unbroken spheroplasts by centrifugation ( 3 times 1 minute at $500 \mathrm{~g}$ ). Microscopic control confirmed that no unbroken cells were left in the lysates. $1.5 \mathrm{ml}$ of each lysate was loaded on separate sucrose density gradients (Schröder et al., 1995) and spun at $4^{\circ} \mathrm{C}$ in a Beckman SW40 rotor at $37,000 \mathrm{rpm}$ for 2:30 hours. $1 \mathrm{ml}$ fractions were taken and the pellet was resuspended in the last fraction which was adjusted to $1 \mathrm{ml}$ with $\mathrm{dH}_{2} \mathrm{O}$. Each fraction was mixed with $1 \mathrm{ml}$ SDS-PAGE sample buffer ( $8 \mathrm{M}$ urea, $50 \mathrm{mM}$ Tris- $\mathrm{HCl}, \mathrm{pH}$ 8.0, 2\% SDS, $0.1 \mathrm{mg} / \mathrm{ml}$ bromphenol blue) and incubated at $50^{\circ} \mathrm{C}$ for 10 minutes prior to analysis by SDSPAGE and immunoblotting.

\section{RESULTS}

To monitor the cellular fate of the $v$-SNAREs Bos1p and Bet1p we tagged the $\mathrm{C}$ terminus of Bos $1 \mathrm{p}$ and Bet1p with a 41 amino acid extension as was done previously with $\operatorname{Sec} 22 \mathrm{p}$ (Ballensiefen et al., 1998) (Fig. 1). The tag includes a $c$-myc epitope, a Kex $2 p$ cleavage site and the 13 residue long $\alpha$-factor. This tag allowed us to detect the hybrid protein with two different antibodies: anti-c-myc and anti- $\alpha$-factor. Using this approach we could follow the arrival of the constructs in the late Golgi where $\alpha$-factor is cleaved off by the late Golgi protease Kex2p. The cleavage can be visualized using SDSpolyacrylamide gel electrophoresis and immunoblotting (Ballensiefen et al., 1998). The cleaved protein is no longer recognized by anti- $\alpha$-factor antibody.

To make sure that the luminal extension of the proteins did not impair their function, temperature sensitive bosl ( $\sec 32-1)$ (Wuestehube et al., 1996) and bet $1-1$ mutants (Newman et al., 1990) were transformed with plasmids bearing the BOSI- $\alpha$ and

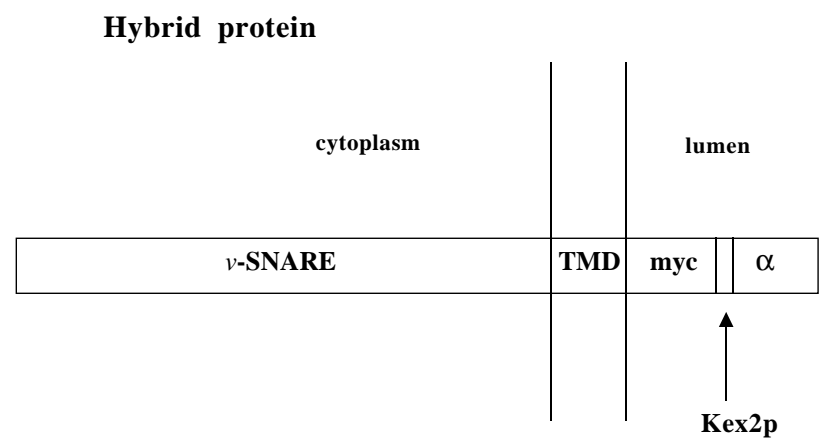

Fig. 1 Scheme of the hybrid protein including the c-myc epitope fused to Bos $1 p$ or Bet1p, the Kex $2 p$-cleavage site and $\alpha$-factor tag. Note that the overall extension of the wild-type proteins is only 41 residues. The Kex $2 p$ trimmed Bos $1-\alpha$ and Bet1- $\alpha$ can be detected by western blot analysis using the anti-Bos $1 \mathrm{p}$ (anti-Bet1p) serum or the anti-c-myc antibody but not with antibodies specific for the $\alpha$-factor.
BET1- $\alpha$ constructs, respectively. Transformed cells were able to grow at non-permissive temperature $\left(37^{\circ} \mathrm{C}\right)$ (data not shown). The hybrid proteins were put under control of the $G A L 1$ promoter to assure a sufficient production of the proteins for indirect immunofluorescence analysis. In addition, the use of the glucose-repressible GAL1 promoter allowed us to carry out non-radioactive 'pulse-chase' experiments. The fate of the protein, which had been produced during incubation in galactose medium (pulse) could be traced after shift to glucose medium (chase).

\section{Bos $1-\alpha$ is more rapidly cleaved by Kex $2 p$ in COPI mutants compared to wild-type cells}

It was shown that at steady state the ER retention of Sec $22 p$ is affected by mutations in some COPI components (Ballensiefen et al., 1998). To investigate the targeting of Bos $1-\alpha$ and to see whether its localization also depends on COPI, we transformed sec $21-1$ and sec27-1 mutant cells as well as the corresponding wild-type cells with these constructs.

Cells were incubated overnight in galactose-containing medium (pulse). The production of the hybrid proteins was stopped by transferring the cells into glucose-containing medium (chase). Incubation during both pulse and chase time was done at $25^{\circ} \mathrm{C}$ because $\sec 21-1$ and $\sec 27-1$ exhibit the recycling defect already at permissive temperature (Letourneur et al., 1994). Chase was performed for 0, 1 and 2 hours. Immunoblot analysis was used to determine the rate of Kex2pcleavage in these strains. In contrast to Sec22- $\alpha$ and Bet1- $\alpha$ (see below), we could not detect a size difference between cleaved and uncleaved Bos $1-\alpha$, the reason for which was not clear. In order to distinguish cleaved from uncleaved Bos1 hybrid proteins either anti-c-myc or anti- $\alpha$-factor antibodies were applied. Only the uncleaved protein can be detected with the anti- $\alpha$-factor antibody whereas the anti- $c$-myc antibody recognizes both cleaved and uncleaved forms of the protein.

Using the anti-c-myc antibody, Bos $1-\alpha$ protein is detectable at all time points in both wild-type and sec21-1 mutant cells (Fig. 2). However, even in wild-type cells the amount of uncleaved Bos $1-\alpha$ recognized by $\alpha$-factor antibody decreased with time indicating that the protein had reached the late Golgi compartment. However, cleavage proceeded much faster in the isogenic sec 21-1 mutant strain. Kex2p-cleavage was complete

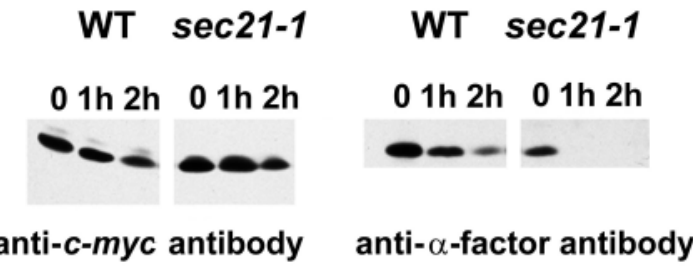

Fig. 2 Immunoblot analysis of Bos1- $\alpha$ protein produced in sec21-1 mutant (S21P4-9A) and corresponding wild-type cells (S21P4-6A). Cells were grown overnight in galactose-containing medium at $25^{\circ} \mathrm{C}$ to induce hybrid protein production (pulse) and then were transferred to glucose-containing medium to stop synthesis of Bos1- $\alpha$ (chase). Incubation was continued at $25^{\circ} \mathrm{C}$. Aliquots of cells were taken after 0 minutes, 1 hour and 2 hours of incubation in glucose-containing medium. Bos $1-\alpha$, cleaved and uncleaved by Kex $2 p$, was detected by polyclonal anti-c-myc antibody. Anti- $\alpha$-factor antibody was used to detect only uncleaved hybrid protein. 
A
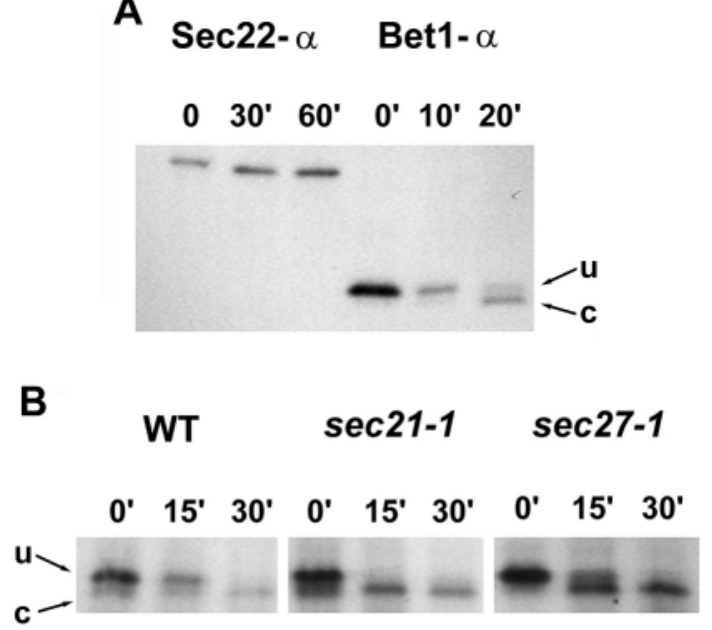

Fig. 3 (A) Pulse-chase experiment with wild-type cells (S21P4-6A) producing Sec22- $\alpha$ or Bet1- $\alpha$. The cells were treated as described in Materials and Methods. Cells were labeled for 7 minutes with Trans $\left[{ }^{35} \mathrm{~S}\right]$-label. Aliquots were taken at 0,30 and 60 minutes of chase time for Sec22- $\alpha$ and at 0,10 and 20 minutes for Bet1- $\alpha$. A mixture of anti-c-myc and anti- $\alpha$-factor antibodies was used to precipitate uncleaved (u) as well as cleaved (c) protein. (B) Pulsechase experiment to follow the cleavage of Bet1- $\alpha$ in wild-type cells (S21P4-6A), and the COPI mutants sec21-1 (S21P4-9A) and sec27-1 (S27P4-9C). Pulse time was 5 minutes. Aliquots were taken at 0,15 and 30 minutes of chase time.

within 1 hour. The same result was obtained using a sec27-1 mutant (data not shown). Thus the rate of Kex2p-cleavage is higher in the COPI mutants than in wild-type cells.

\section{Bet1- $\alpha$ reaches the late Golgi faster than Sec22- $\alpha$ and Bos1- $\alpha$}

Initial western blotting analysis showed that the processing of Bet1- $\alpha$ was extremely fast when the hybrid protein was expressed under the same conditions described for Bos1- $\alpha$ and Sec22- $\alpha$. Even at the beginning of the chase the protein was hardly detectable by $\alpha$-factor antibody in the wild type and not recognized at all in the mutant (data not shown). Therefore, radioactive pulse-chase experiments were performed to follow the cleavage of Bet1- $\alpha$. The comparison of Bet1- $\alpha$ with Sec22$\alpha$ in wild-type cells showed that after 7 minutes of pulse-time and 20 minutes of chase more than $50 \%$ of Bet $1-\alpha$ was cleaved by Kex2p (Fig. 3A). However, no cleavage was observed for Sec22- $\alpha$ even after 1 hour of chase-time. Thus Bet1- $\alpha$ translocates to the late Golgi from earlier cellular compartments more rapidly than Sec22- $\alpha$ and Bos1- $\alpha$. The coatomer mutant sec $21-1$ showed a slightly increased rate of Bet1- $\alpha$ processing by Kex $2 p$ (Fig. 3B). In this experiment the pulse-time was 5 minutes. Cleavage occurs slightly faster than in wild-type cells (compare 15 minute time-points; Fig. 3B). The sec27-1 mutant cells show a rate of cleavage comparable with that of wild-type cells. In both wild-type and COPI mutant cells most of the protein is processed by Kex $2 p$ after 30 minutes of chase-time.

\section{Bos1- $\alpha$ and Bet1- $\alpha$ localize to different parts of the secretory pathway}

Indirect immunofluorescence microscopy was used to
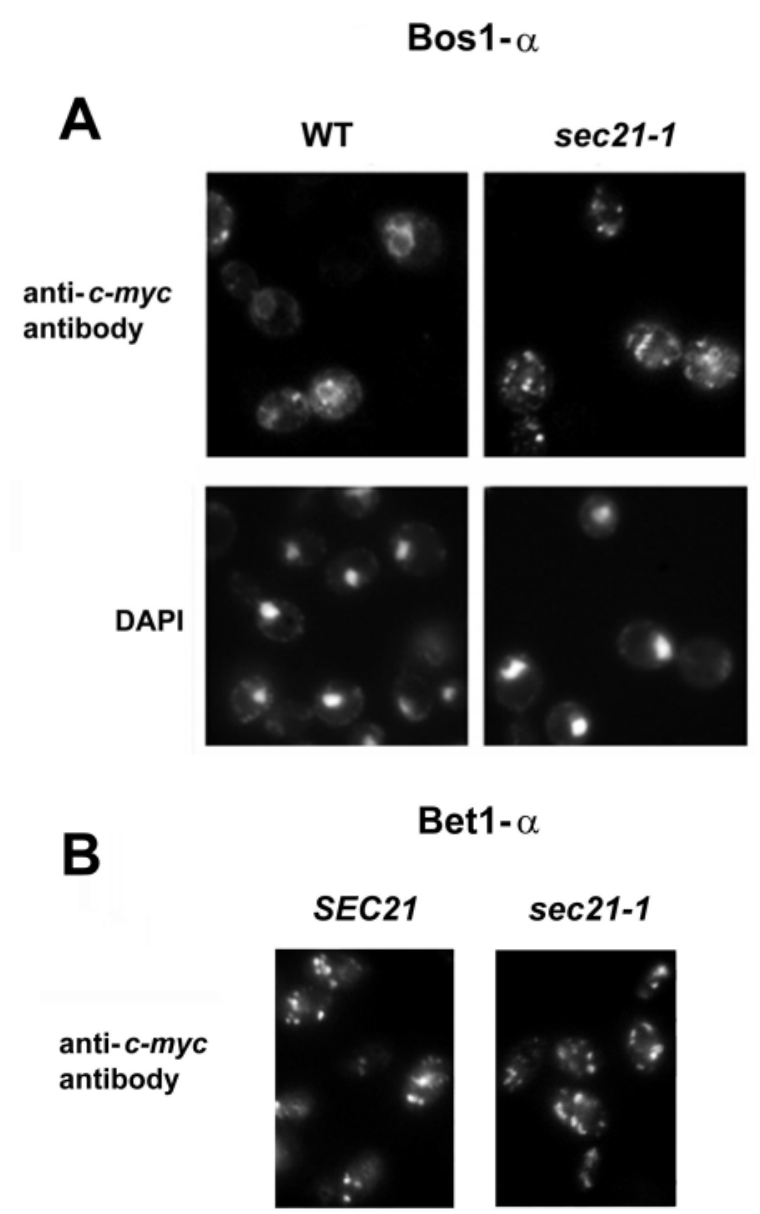

Fig. 4 Indirect immunofluorescence analysis to determine the cellular localization of Bos1- $\alpha$ (A) and Bet1- $\alpha$ (B) in wild-type SEC21 (S21P4-6A) cells and an isogenic sec21-1 (S21P4-9A) mutant strain. The hybrid protein synthesis was induced by incubating cells in galactose-containing medium overnight. Then cells were kept at the same temperature for 2 hours in glucose-containing medium to stop the synthesis of hybrid protein and treated as described in Materials and Methods. Incubation temperature was $25^{\circ} \mathrm{C}$ for both pulse and chase time. Cells were stained with anti-c-myc monoclonal antibody and Cy3-conjugated secondary antibody. DAPI staining was used to localize the nuclei.

determine the localization of Bos1- $\alpha$ and Bet1- $\alpha$ in wild-type and sec21-1 cells. It is important to note that aliquots of the same cell culture expressing Bos1- $\alpha$ used for immunoblot analysis (see above) were also taken for immunofluorescence. Thus the growth conditions $\left(25^{\circ} \mathrm{C}\right.$, overnight pulse in galactose containing medium, two hours of chase in glucose-containing medium) were identical for both experiments. Following this protocol we could determine the distribution of Bos1- $\alpha$ which had already passed through the late Golgi compartment as shown by immunoblot analysis. At the same time, we excluded a contribution of newly synthesized hybrid protein to the immunofluorescence pattern. Bos1- $\alpha$ and Bet1- $\alpha$ were visualized by anti-c-myc antibody which does not recognize constitutively produced endogenous Bos $1 \mathrm{p}$ and Bet1p.

In yeast, the ER stained by immunofluorescence appears as a ring around the nucleus (Rose et al., 1989; Preuss et al., 1992) while the Golgi staining gives a punctuate pattern (Franzusoff 
A
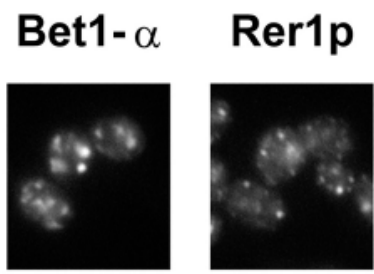

anti-c-myc antibody

anti-Rer1p antibody

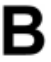

Bet1-HA Och1-c-myc

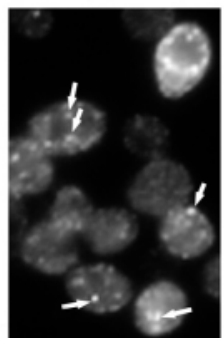

anti-HA antibody

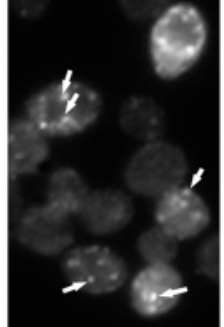

anti-c-myc antibody
Fig. 5 (A) Double indirect immunofluorescence analysis shows no colocalization of Bet $1 p$ and the middle Golgi marker, Rer1p at steady-state conditions. Cells were incubated overnight in galactosecontaining medium. Bet1p was c-myc tagged and detected by anti- $c$ myc monoclonal antibody. Rerlp was recognized by anti-Rer1p polyclonal antibody. Cy3-conjugated anti-mouse and Cy2conjugated anti-rabbit secondary antibodies were used to bind to anti-c-myc and anti-Rer1p, respectively. (B) Double indirect immunoflourescense microscopy to demonstrate colocalization of Bet1p and the early Golgi marker Och1p at steady-state conditions. Cells were incubated overnight in galactose-containing medium. Betlp was HA-tagged and detected by anti-HA polyclonal antibody. Och1p was $c$ - $m y c$-tagged and recognized by anti-c-myc monoclonal antibody. Cy3-conjugated anti-rabbit and Cy2-conjugated antimouse secondary antibodies were used to bind to anti-HA and anti$c-m y c$, respectively.

et al., 1991; Redding et al., 1991; Antebi and Fink, 1992). To visualize the nuclei, cells were stained with the fluorescent dye DAPI. Fig. 4A (left panel) shows an ER localization of Bos1- $\alpha$ in wild-type cells after two hours of glucose-chase. At the same time-point, immunoblot analysis revealed that a considerable amount of Bos1- $\alpha$ was already cleaved by the late Golgi protease Kex2p. Taken together these results indicate that there is an efficient recycling system that brings Bos $1-\alpha$ back to the ER from later compartments. The sec21-1 mutant shows only a punctuate pattern indicating that Bos $1-\alpha$ is mislocalized to the Golgi (Fig. 4A, right panel). The same results were obtained for a pair of isogenic wild-type and sec27-1 cells (data not shown). Thus, Bos1- $\alpha$ requires functional COPI components to be recycled from the Golgi to the ER.

Different from Bos $1-\alpha$, Bet $1-\alpha$ is localized to the Golgi in wild-type cells (Fig. 4B, left panel). Double immunofluorescence under steady-state conditions in wildtype cells was used to ditinguish between different subcompartments of the Golgi. Bet1- $\alpha$ did not co-localize with
Emp47p
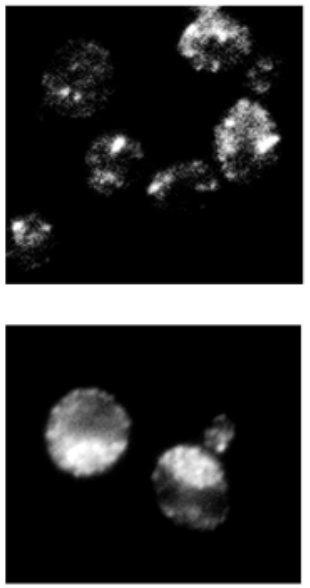

Bet1- $\alpha$
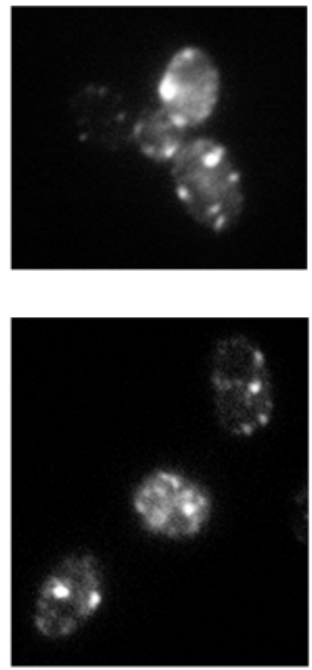

DAPI
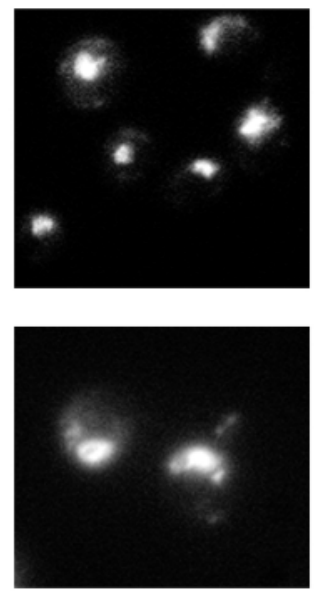

$35{ }^{\circ} \mathrm{C}$

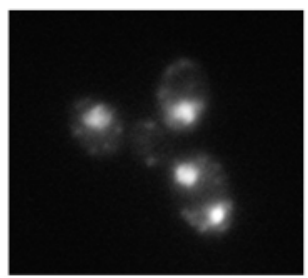

Fig. 6 Cellular distribution of Bet1- $\alpha$ in sec23-1 (589-3C) mutant and wild-type cells using indirect immunoflourescence analysis. Cells were incubated overnight in galactose-containing medium at $25^{\circ} \mathrm{C}$, hybrid protein production was stopped by cycloheximide and then cells were incubated in this medium additionally for one hour at non-permissive $\left(35^{\circ} \mathrm{C}\right)$ or permissive $\left(25^{\circ} \mathrm{C}\right)$ temperature. Cells were stained with anti-c-myc monoclonal or anti-Emp47p antibody and Cy3-conjugated secondary antibody. DAPI staining shows the location of the nuclei.

the middle Golgi protein Rer1p (Boehm et al., 1997) (Fig. 5A). But the staining pattern of Bet1p and the early Golgi marker Och1p (Harris and Waters, 1996) were almost identical (Fig. 5B). A $c$-myc-tagged version of Och1p expressed at a high expression level was used to allow detection in immunofluorescence. For this experiment hemagglutinin (HA)-tagged, Betlp was employed and anti-HA antibody was applied to detect this protein.

The results presented in Fig. 4B (right panel) show that Bet $1-\alpha$ stays in the Golgi in sec $21-1$ mutant cells unlike some Golgi proteins such as Emp47, which mislocalize to the vacuole under the same conditions (Lewis and Pelham, 1996; 
Schröder-Köhne et al., 1998). However, as shown for the Golgi proteins such as Emp47p (Schröder et al., 1995), Erd2p (Lewis and Pelham, 1996) and Sed5p (Wooding and Pelham, 1998), Bet1p may also cycle between the Golgi and the ER. Recycling can be demonstrated by trapping recycled proteins in the ER utilizing an ER budding COPII mutant (Schröder et al., 1995). Therefore, we transformed sec23-1 (COPII mutant) cells with a plasmid carrying BETl- $\alpha$. For unknown reasons the glucose shut-off of Bet1- $\alpha$ expression was not complete in this particular mutant as we could detect newly synthesized protein accumulated in the ER (data not shown). Therefore, after overnight incubation in galactose-containing medium at $25^{\circ} \mathrm{C}$, the hybrid protein production was stopped by the addition of cycloheximide. Cells were incubated for 5 more minutes at $25^{\circ} \mathrm{C}$ and then for one hour at nonpermissive temperature $\left(35^{\circ} \mathrm{C}\right)$ to block the anterograde transport between the ER and the Golgi. sec $23-1$ cells treated with cycloheximide and incubated at $25^{\circ} \mathrm{C}$ for one hour were used as a control. For immunofluorescence analysis cells were stained for Bet1- $\alpha$ or Emp47p. sec23-1 cells at both permissive and non-permissive temperatures displayed a punctuate Golgi immunofluorescence pattern for Bet1- $\alpha$ (Fig. 6). As a positive control for Golgi-ER recycling we used the Golgi protein Emp47p (Schröder et al., 1995). In contrast to Bet1- $\alpha$ Emp47p displayed the expected ER pattern at $35^{\circ} \mathrm{C}$. Experiments performed with endogenous Bet1p protein (see below) supported this observation.

\section{Localization of endogenous Bos1p and Bet1p}

To verify that the localization of Bos $1-\alpha$ and Bet $1-\alpha$ reflects the behavior of the wild-type proteins we investigated the distribution of the endogenous proteins using fractionation on sucrose-density gradients (Fig. 7A). sec23-1 mutant cells were incubated in presence of cycloheximide at either permissive $\left(25^{\circ} \mathrm{C}\right)$ or non-permissive $\left(35^{\circ} \mathrm{C}\right)$ temperature for one hour. Cell lysates were prepared and applied to sucrose gradients (see Materials and Methods). The distribution of the Golgi protein Emp47p and the ER protein Kar2p was determined as a control.
As shown in Fig. 7B, the distribution of endogenous Bos $1 \mathrm{p}$ and Bet1p determined by sucrose gradient fractionation correlates very well with that obtained for Bos $1-\alpha$ and Bet1$\alpha$ hybrid proteins by immunofluorescence: the majority of Bet1p is found in the Golgi at both permissive and nonpermissive temperatures, whereas Bos $1 \mathrm{p}$ is localized predominantly in the ER under the same conditions. In contrast, Emp47p redistributed from Golgi fractions at $25^{\circ} \mathrm{C}$ to the ER fractions at $35^{\circ} \mathrm{C}$ as described previously (Schröder-Köhne et al., 1998).

\section{DISCUSSION}

This work provides information about the targeting of $v$-SNAREs involved in the anterograde transport between the ER and the Golgi. Importantly, these proteins exhibit differences in their dynamics and steady-state localization. This could reflect different roles they play in the early step of vesicular transport. Two assays were employed to investigate the targeting of Bos1p and Bet1p. Kex2p-dependent cleavage of Bos1- $\alpha$ and Bet1- $\alpha$

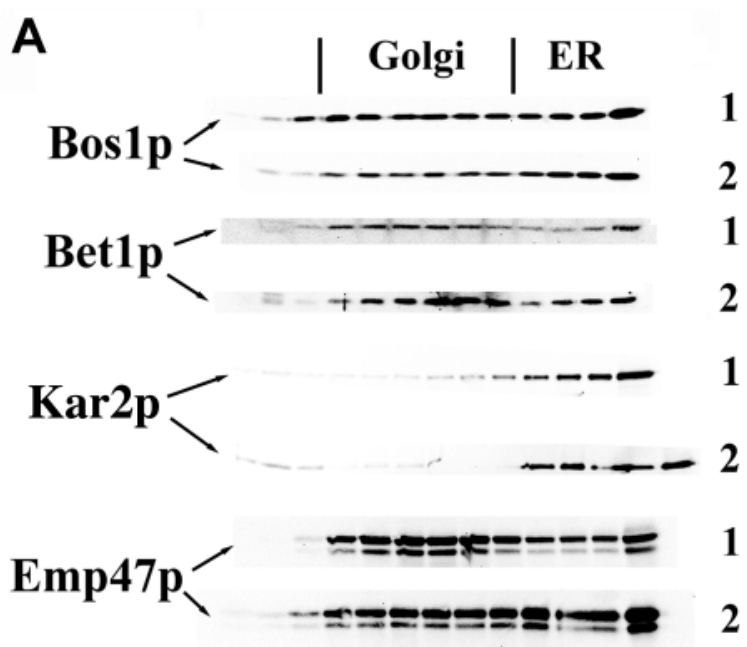

B

Fig. 7 (A) Cellular distribution of Bos1p, Bet1p, Kar2p and Emp47p on sucrosedensity gradient in sec 23-1 mutant (SSY78) at permissive (1) and nonpermissive (2) temperature (see Materials and Methods for details). SSY78 expresses a $m y c$-tagged version of Emp47p (migrating more slowly) in addition to endogenous Emp47p. Both proteins fractionate identically (Schröder et al., 1995). (B) Evaluation of proteins' distribution using Lumi-Imager software. Distribution of each protein is depicted by two adjacent bars: percentage of this protein in the Golgi $(\mathrm{G})$ and in the ER (E). Left panel of the chart shows the distribution at the $25^{\circ} \mathrm{C}$ and the right one at $35^{\circ} \mathrm{C}$.
Golgi-ER protein distribution in sec23-1 cells

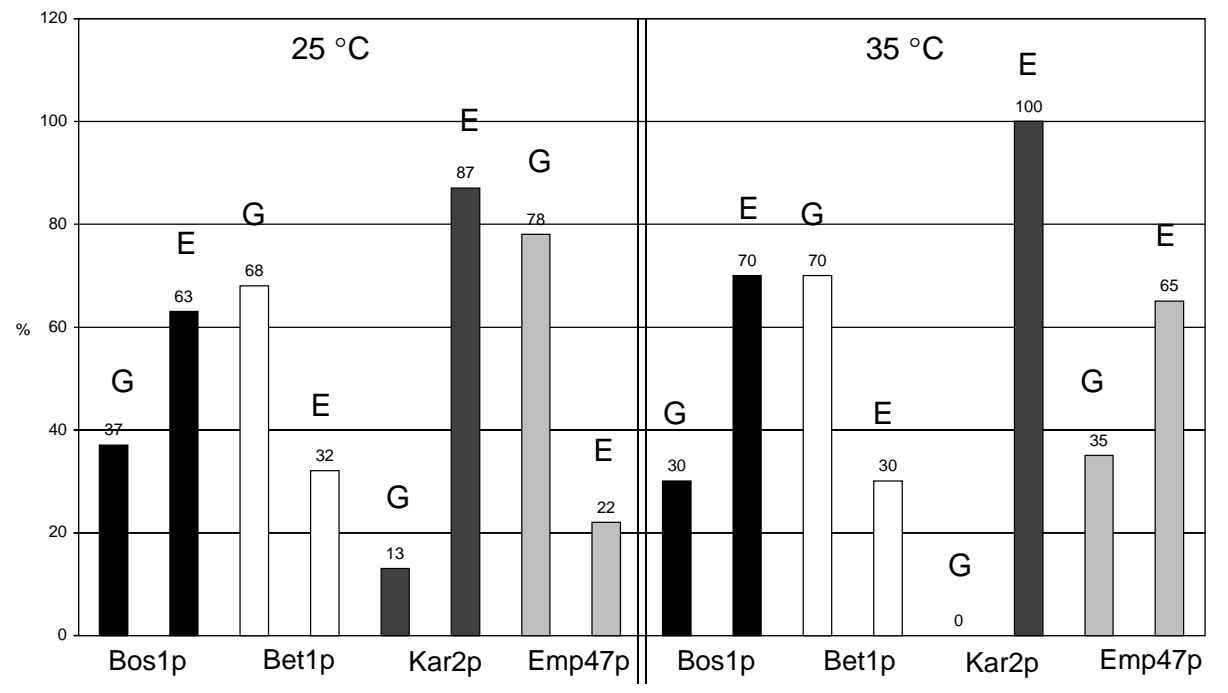


was used to monitor the transport of the tagged protein to the late Golgi compartment. Bos1- $\alpha$, when expressed under the control of the GAL promoter, reaches the late Golgi in wildtype cells within the timeframe of hours. However, immunofluorescence microscopy demonstrates that Bos1- $\alpha$ is located in the ER, even though most of the protein had been cleaved by Kex $2 p$. The observation suggests the presence of a retrieval system for Bos1- $\alpha$ that brings it back to the ER. This recycling apparently depends on COPI components, which is indicated by Kex $2 \mathrm{p}$ cleavage being faster in sec $21-1$ and sec27-1 mutants than in wild-type cells. Furthermore, immunofluorescence analysis showed the accumulation of Bos1$\alpha$ in the Golgi of these mutants. In this respect, it is interesting to note that Bos $1 p$ does apparently not follow the secretory pathway to the vacuole as it has been observed for the di-lysine protein Emp47p (Schröder-Köhne et al., 1998).

Data obtained by immunofluorescence and subcellular fractionation by sucrose-density gradients suggest that Bet1p is Golgi-resident and does not recycle. However, a slightly increased rate of Kex $2 p$ processing of the Bet1- $\alpha$ in sec $21-1$ mutant cells may point at a very slow recycling of Bet1p, which may not have been detectable by the other experimental approaches mentioned above. However, there was no difference in rates of Kex $2 p$ cleavage between wild type and another COPI-mutant strain, sec27-1.

The targeting of Bos $1-\alpha$ is very similar to that of another $v$-SNARE, Sec22p (Ballensiefen et al., 1998). Both proteins are localized to the ER and follow the same path for recycling as the di-lysine-tagged transmembrane proteins (Letourneur et al., 1994), multiple membrane spanning proteins like Erd2p and Rer1p and the proteins that require the latter two for retrieval (Lewis and Pelham, 1996; Boehm et al., 1997; Sato et al., 1997). However, these two proteins may play different roles in the trafficking between the ER and the Golgi. Bos1p, as demonstrated by in vitro experiments (Spang and Schekman, 1998), is required only for forward transport whereas Sec22p is implicated only in retrograde transport.

Bet1p dynamics resemble that of another Golgi protein, mannosyltransferase Och1p that colocalizes very well with Bet1p. Harris and Waters (1996) showed that chimeras derived from Och $1 p$ are cleaved by Kex $2 p$ with a half time of about 5 minutes while the steady state localization of Och1p and its tagged version is the early Golgi. To keep the gradient of Och1p across the Golgi, the fast forward transport must be counteracted by rapid recycling. However, it is still not clear whether Och1p recycles between the Golgi and the ER. Bet1p is not the only example of a Golgi-resident protein that does apparently not recycle to the ER. Previously, this was shown for the yeast Golgi protein Pmr1p (Schröder et al., 1995).

Data presented in this work for the cellular localization of Bet $1 \mathrm{p}$ and Bos $1 \mathrm{p}$ in yeast indicate that Bet1p may extend further into the Golgi than Bos1p or Sec22p. This is in contrast to the observations made by Hay et al. (1998) for mammalian cells where the Bos $1 \mathrm{p}$ homologue membrin penetrates further into the secretory pathway than rbet1 and rsec22b. The situation in mammalian cells is complicated since ER-Golgi transport involves the passage of cargo through an intermediate compartment (IC). There is evidence that ER-Golgi SNAREs recycle through the ER (Wooding and Pelham, 1998; Rowe et al., 1998; Ballensiefen et al., 1998; Hay et al., 1998). SNARE proteins may function at different sites along the mammalian
ER-IC-Golgi route (Zhang et al., 1997; Hay et al., 1998). In yeast there is no evidence for the existence of an intermediate compartment.

According to a recent in vitro study presented by Spang and Schekman (1998) Bos1p in yeast may function primarily in anterograde transport while Betlp may be required for both anterograde and retrograde transport. Therefore, it is surprising that Bet1p is more rapidly transported to the late Golgi than Bos1p. However, the steady-state localization may not reflect the actual site of function. The fact that Bet1p accumulates in the Golgi may reflect a more efficient packaging into COPII vesicles as indicated by the observation that Bet1p binds directly to the COPII complex while Bos1p binds less efficiently and Sec22p did not bind at all (Springer and Schekman, 1998). In accordance with the results obtained by Newman et al. (1992) who used fractionation experiments, we also found that a part of Bet1p cosediments with the ER fraction. However, a most of the protein $(68 \%)$ cofractionated with Golgi markers.

Recycling of $v$-SNAREs via COPI vesicles is necessary to use these proteins for further rounds of vesicle fusion. The next question we would like to address in this respect is whether Bos $1 p$ and Sec22p interact directly with COPI components or, as with other proteins recycling to the ER, a receptor or adapter is required for sorting and packaging of these proteins into COPI vesicles (Semenza et al., 1990; Boehm et al., 1997).

We are very grateful to Wolfgang Ballensiefen, Rainer Duden, Mike Lewis, François Letourneur and M. Gerard Waters for providing plasmids and yeast strains. We thank Wolfgang Ballensiefen, Tanja Neumann for critical comments on the manuscript. We also thank Johannes Boehm and Mustafa Benli for supplying antibodies and Hannegret Frahm for technical assistance. This work was in part supported by the Deutsche Forschungsgemeinschaft (SFB 523) and by a DFG-fellowship and grant (Schr561/1-3+/1-4) to S.S.-K.

\section{REFERENCES}

Antebi, A. and Fink, G. R. (1992). The yeast $\mathrm{Ca}^{2+}$-ATPase homologue, $P M R 1$, is required for normal Golgi function and localizes in a novel Golgilike distribution. Mol. Biol. Cell 3, 633-654.

Aridor, M., Bannykh, S. I., Rowe, T. and Balch, W. E. (1995). Sequential coupling between COPII and COPI vesicle coats in endoplasmic reticulum to Golgi transport. J. Cell Biol. 131, 875-893.

Aridor, M., Weissnan, J., Bannykh, S. I., Nuoffer, C. and Balch, W. E. (1998). Cargo selection by the COPII budding machinary during export from the ER. J. Cell Biol. 141, 61-70.

Ballensiefen, W., Ossipov, D. and Schmitt, H. D. (1998). Recycling of yeast $v$-SNARE Sec22p involves COPI-proteins and the ER transmembrane proteins Ufe1p and Sec20p. J. Cell Sci. 111, 1507-1520.

Barlowe, C., Orci, L., Yeung, T., Hosobuchi, M., Hamamoto, S., Salama, N., Rexach, M. F., Ravazzola, M., Amherdt, M. and Schekman, R. (1994). COPII: a membrane coat formed by Sec proteins that drive vesicle budding from the endoplasmic reticulum. Cell 77, 895-907.

Bednarek, S., Ravazzola, M., Hosobuchi, M., Amherdt, M., Perrelet, A., Schekman, R. and Orci, L. (1995). COPI-coated and COPII-coated vesicles bud directly from the endoplasmic reticulum in yeast. Cell $\mathbf{8 3}, 1183$ 1196.

Boehm, J., Ulrich, H. D., Ossig, R. and Schmitt, H. D. (1994). Kex2dependent invertase secretion as a tool to study the targeting of transmembrane proteins which are involved in ER-Golgi transport in yeast. EMBO J. 13, 3696-3710.

Boehm, J., Letourneur, F., Ballensiefen, W., Ossipov, D., Démollière, C. and Schmitt, H. D. (1997). Sec12p requires Rer1p for sorting to coatomer (COPI)-coated vesicles and retrieval to the ER. J. Cell Sci. 110, 991-1003. 
Cosson, P., Démollière, C., Hennecke, S., Duden, R. and Letourneur F. (1996). $\delta$-COP and $\zeta$-COP, two coatomer subunits homologous to clathrinassociated proteins, are involved in ER retrieval. EMBO J. 15, 1792-1798.

Dascher, C., Ossig, R., Gallwitz, D. and Schmitt, H. D. (1991). Identification and structure of four yeast genes (SLY) that are able to suppress the functional loss of YPT1, a member of the ras-superfamily. Mol. Cell. Biol. 11, 872-885.

Duden, R., Hosobuchi, M., Hamamoto, S., Winey, M., Byers, B. and Schekman, R. (1994). Yeast $\beta$ - and $\beta^{\prime}$-coat proteins (COP). Two coatomer subunits essential for endoplasmic reticulum-to-Golgi protein traffic. J. Biol. Chem. 269, 24486-24495.

Duden, R., Kajikawa, L., Wuestehube, L. and Schekman, R. (1998). ع-COP is a structural component of coatomer that functions to stabilize $\alpha$-COP. EMBO J. 17, 985-995.

Evan, G. J., Lewis, G. K., Ramsay, G. and Bishop, J. M. (1985). Isolation of monoclonal antibodies specific for human c-myc proto-oncogene product. Mol. Cell. Biol. 5, 3610-3616.

Franzusoff, A., Redding, K., Crosby, J., Fuller, R. S. and Schekman, R. (1991). Localization of components involved in protein transport and processing through the yeast Golgi-apparatus. J. Cell Biol. 112, 27-37.

Gaynor, E. C. and Emr, S. D. (1997). COPI-independent anterograde transport: cargo-selective ER to Golgi protein transport in yeast COPI mutants. J. Cell Biol. 136, 789-802.

Götte, M. and Fischer von Mollard, G. (1998). A new beat for the SNARE drum. Trends Cell Biol. 8, 215-218.

Hardwick, K. G. and Pelham, H. R. B. (1992). SED5 encodes a 39-kD integral membrane protein required for vesicular transport between the ER and the Golgi complex. J. Cell Biol. 119, 513-521.

Harris, S. L. and Waters, M. G. (1996). Localization of a yeast early Golgi mannosyltransferase, Och1p, involves retrograde transport. J. Cell Biol. 132, 985-998.

Hay, J. C., Klumperman, J., Oorschot, V., Steegmaier, M., Kuo C. S. and Scheller R. H. (1998). Localization, dynamics, and protein interactions reveal distinct roles for ER and Golgi SNAREs. J. Cell Biol. 141, 14891502.

Hong, W. (1998). Protein transport from the endoplasmic reticulum to the Golgi apparatus. J. Cell Sci. 111, 2831-2839.

Kuehn, M. J., Herrmann, J. M. and Schekman, R. (1998). COPII-cargo interactions direct protein sorting into ER-derived transport vesicles. Nature 391, 187-190.

Letourneur, F., Gaynor, E. C., Hennecke, S., Démollière, C., Duden, R., Emr, S. D., Riezman, H. and Cosson, P. (1994). Coatomer is essential for retrieval of di-lysine-tagged proteins to the endoplasmic reticulum. Cell 79, 1199-1207.

Lewis, M. J. and Pelham, H. R. B. (1996). SNARE-mediated retrograde traffic from the Golgi-complex to the endoplasmic reticulum. Cell 85, 205215.

Lewis, M. J., Raynor, J. C. and Pelham, H. R. B. (1997). A novel SNARE complex implicated in vesicle fusion with the endoplasmic reticulum. EMBO J. 16, 3017-3024.

Mayer, A., Wickner, W. and Haas, A. (1996). Sec18p (NSF)-driven release of Sec $17 \mathrm{p}(\alpha-$ SNAP) can precede docking and fusion of yeast vacuoles. Cell 85, 83-94.

Newman, A. P., Shim, J. and Ferro-Novick, S. (1990). BET1, BOS1, and $S E C 22$ are members of a group of interacting yeast genes required for transport from the endoplasmic reticulum to the Golgi complex. Mol. Cell. Biol. 10, 3405-3414.

Newman, A. P., Groesch, M. E. and Ferro-Novick, S. (1992). Bos1p, a membrane-protein required for ER to Golgi transport in yeast, copurifies with the carrier vesicles and with Betlp and the ER membrane. EMBO J. 11, 3609-3617.

Orci L., Stamnes, M., Ravazzola, M., Amherdt, M., Perrelet, A., Söllner, T. H. and Rothman, J. E. (1997). Bidirectional transport by distinct populations of COPI-coated vesicles. Cell 90, 335-349.

Otto, H., Hanson, P. I. and Jahn, R. (1997). Assembly and disassembly of a ternary complex of synaptobrevin, syntaxin, and SNAP-25 in the membrane of synaptic vesicles. Proc. Nat. Acad. Sci. USA 94, 6197-6201.

Preuss, D., Mulholland, J., Franzusoff, A., Segev, N. and Botstein, D. (1992). Characterization of the Saccharomyces Golgi complex through the cell-cycle by immunoelectron microscopy. Mol. Biol. Cell 3, 789-803.

Redding, K., Holcomb, C. and Fuller, R. S. (1991). Immunolocalization of Kex2 protease identifies a putative late Golgi compartment in the yeast Saccharomyces cerevisiae. J. Cell Biol. 113, 527-538.
Rose, M. D., Misra, L. M. and Vogel, J. P. (1989). KAR2, a karyogamy gene, is the yeast homolog of the mammalian BiP/GRP78 gene. Cell 57, 12111221.

Rothman, J. E. (1994). Mechanism of intracellular protein-transport. Nature 372, 55-63.

Rowe, T., Aridor, M., McCaffery, J. M., Plutner, H., Nuoffer, C. and Balch, W. E. (1996). COPII vesicles derived from mammalian endoplasmic reticulum microsomes recruit COPI. J. Cell Biol. 135, 895-911.

Rowe, T., Dascher, C., Bannykh, S., Plutner, H and Balch, W. E. (1998) Role for vesicle-associated syntaxin 5 in the assembly of the pre-Golgi intermediates. Science 279, 696-700.

Sato, K., Sato, M. and Nakano, A. (1997). Rer1p as common machinery for the endoplasmic reticulum localization of membrane proteins. Proc. Nat. Acad. Sci. USA 94, 9693-9698.

Scales, S. J., Pepperkok, R. and Kreis, T. E. (1997). Visualization of ER-toGolgi transport in living cells reveals a sequential mode of action for COPII and COPI. Cell 90, 1137-1148.

Schekman, R. and Orci, L. (1996). Coat proteins and vesicle budding Science 271, 1526-1533.

Schröder, S., Schimmöller, F., Singer-Krüger, B. and Riezman, H. (1995) The Golgi-localization of yeast Emp47p depends on its di-lysine motif but is not affected by the ret1-1 mutation in $\alpha$-COP. J. Cell Biol. 131, 895-912.

Schröder-Köhne, S., Letourneur, F. and Riezman, H. (1998). $\alpha$-COP can discriminate between distinct, functional dy-lysine signals in vitro and regulates access into retrograde transport. J. Cell Sci. 111, 3458-3470.

Semenza, J. C., Hardwick, K. G., Dean, N. and Pelham, H. R. B. (1990). $E R D 2$, a yeast gene required for the receptor-mediated retrieval of luminal ER proteins from the secretory pathway. Cell 61, 1349-1357.

Shen, S.-H., Chretien, P., Bastien, L. and Slilaty, S. N. (1991). Primary sequence of the glucanase gene from Oerskovia xanthineolytica. J. Biol. Chem. 2, 1058-1063.

Søgaard, M., Tani, K., Ye, R. R., Geromanos, S., Tempst, P., Kirchhausen, T., Rothman, J. E. and Söllner, T. (1994). A rab protein is required for the assembly of SNARE complexes in the docking of transport vesicles. Cell 78, 937-948.

Söllner, T., Whitehart, S. W., Brunner, M., Erdjument-Bromage, H., Geromanos, S., Tempst, P. and Rothman, J. E. (1993). SNAP receptors implicated in vesicle targeting and fusion. Nature 362, 318-324.

Söllner, T. and Rothman, J. E. (1994). Neurotransmission harnessing fusion machinery at the synapse. Trends Neurosci. 17, 344-348.

Spang, A. and Schekman, R. (1998). Reconstitution of retrograde transport from the Golgi to the ER in vitro. J. Cell Biol. 143, 589-599.

Spang, A., Matsuoka, K., Hamamoto, S., Schekman, R. and Orci, L. (1998). Coatomer, Arf1p, and nucleotide are required to bud coat protein complex I-coated vesicles from large synthetic liposomes. Proc. Nat. Acad. Sci. USA 95, 11199-11204.

Springer, S. and Schekman, R (1998). Nucleation of COPII vesicular coat complex by endoplasmic reticulum to Golgi vesicle SNAREs. Science 281, 698-700.

Stamnes, M., Schiavo, G., Stenbeck, G., Söllner, T. H. and Rothman, J. E. (1998). ADP-ribosylation factor and phosphatidic acid levels in Golgi membranes during budding of coatomer-coated vesicles. Proc. Nat. Acad. Sci. USA 95, 13676-13680

Sweet, D. J. and Pelham, H. R. B. (1992). The Saccharomyces cerevisiae SEC20 gene encodes a membrane glycoprotein which is sorted by the HDEL retrieval-system. EMBO J. 11, 423-432.

Waters, M. G., Serafini, T. and Rothman, J. E. (1991). 'Coatomer': a cytosolic protein complex containing subunits of non-clathrin-coated Golgi transport vesicles. Nature 349, 248-251.

Wooding, S. and Pelham, H. R. B. (1998). The dynamics of Golgi protein traffic visualized in living yeast cells. Mol. Biol. Cell 9, 2667-2680.

Wuestehube, L. J., Duden, R., Eun, A., Hamamoto, S., Korn, P., Ram, R. and Schekman, R. (1996). New mutants of Saccharomyces cerevisiae affected in the transport of proteins from the endoplasmic reticulum to the Golgi-complex. Genetics 142, 393-406.

Zhang, T., Wong, S. H., Tang, B. L., Xu, Y., Peter F., Subramaniam, V. N. and Hong, W. (1997). The mammalian protein (rbet1) homologues to yeast Bet $1 \mathrm{p}$ is primarily associated with the pre-Golgi intermediate compartment and is involved in vesicular transport from the endoplasmic reticulum to the Golgi apparatus. J. Cell Biol. 139, 1157-1168.

Yang, B., Gonzalez, L. Jr, Prekeris, R., Steegmaier, M., Advani, R. J. and Scheller, R. H. (1999). SNARE interactions are not selective. J. Biol. Chem. 274, 5649-5653. 